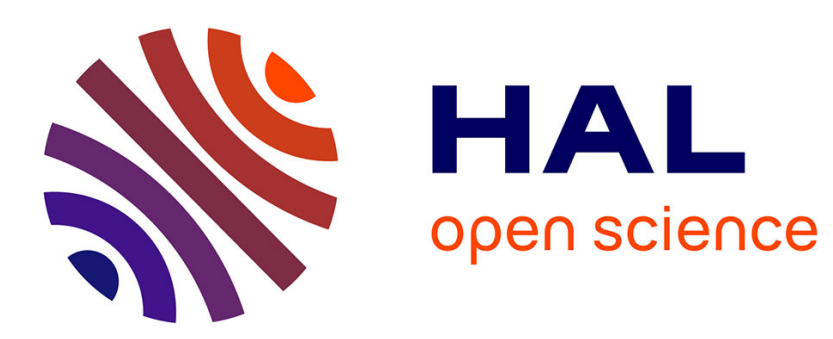

\title{
Formes et dispositifs de reliance pour la construction des identités professionnelles dans le secteur sanitaire et social
}

Pierre Hébrard

\section{- To cite this version:}

Pierre Hébrard. Formes et dispositifs de reliance pour la construction des identités professionnelles dans le secteur sanitaire et social. Revue Phronesis, 2016, 10.7202/1037196ar . hal-01658036

\section{HAL Id: hal-01658036 \\ https://hal.science/hal-01658036}

Submitted on 7 Dec 2017

HAL is a multi-disciplinary open access archive for the deposit and dissemination of scientific research documents, whether they are published or not. The documents may come from teaching and research institutions in France or abroad, or from public or private research centers.
L'archive ouverte pluridisciplinaire HAL, est destinée au dépôt et à la diffusion de documents scientifiques de niveau recherche, publiés ou non, émanant des établissements d'enseignement et de recherche français ou étrangers, des laboratoires publics ou privés. 


\title{
Formes et dispositifs de reliance pour la construction des identités professionnelles dans le secteur sanitaire et social
}

\author{
P. Hébrard LIRDEF, Université de Montpellier
}

(2016). Phronesis, vol. 5/1, 76-83.

Résumé :

Dans les dispositifs de formation professionnelle en alternance, comme ceux mis en œuvre dans le secteur sanitaire et social, le dépassement de l'écart et des tensions entre les temps et les lieux différents de la formation que sont les périodes de stage pratique sur le terrain et les séquences de formation en institut réclame l'instauration d'une coopération entre les acteurs impliqués : personnes en formation, formateurs et tuteurs. Celle-ci, regardée sous l'éclairage du construit de reliance, prend différentes formes et s'appuie sur différents moyens (dispositifs pédagogiques, livrets, portfolios, etc.). L'étude de certains d'entre eux a mis en évidence qu'ils peuvent être situés en référence à deux pôles ayant valeur de paradigmes : l'un se référant à une vision technique de la formation et de l'exercice de la profession (et à une "identité technicienne), l'autre à une approche clinique de la formation et à une "identité clinicienne" des professions concernées.

Ceux qui connaissent mes travaux des cinq dernières années savent que je m'intéresse aux dispositifs de formation en alternance dans les métiers de la formation, de la santé et du travail social, ce que j'appelle les métiers de la relation humaine. Et en particulier à la façon dont ces dispositifs prennent en compte ce qui en constitue le cœur de métier : "les compétences relationnelles et sociales".

En 2010 à l'AREF de Genève, j'avais présenté une première étude centrée sur la réforme de la formation des infirmières en France, ainsi que sur un dispositif de formation de travailleurs sociaux : les techniciennes de l'intervention sociale et familiale (TISF). Cette étude a donné lieu en 2011 à un article dans la revue des sciences de l'éducation de Caen : "L'humanité comme compétence? Une zone d'ombre dans la professionnalisation aux métiers de l'interaction avec autrui" (Hébrard 2011).

J'ai poursuivi ce travail en 2012 avec une approche plus théorique concernant les différents modèles de la compétence sous jacents à ces dispositifs : une première version a été présentée à la "working conference" du ROIP à Barcelone, et une seconde version plus complète en 2013, dans un article de la revue "Les dossiers des sciences de l'éducation" (Hébrard 2013a). J'ai aussi publié en 2013 sur ce thème un article en anglais dans l' European Journal for Research on the Education and Learning of Adults (Hébrard 2013b), et, la même année, une communication dans un symposium à l'AREF.

L'an dernier, j'ai repris ces questions et j'ai tenté de les relier à la notion de "care", et plus 
précisément de "travail du care" (Molinier 2013), dans un chapitre à paraitre (2015) dans un ouvrage collectif co-dirigé par V. Haberey- Knuessi et J-L. Heeb, ouvrage dont le titre devrait être : " Pour une critique de la compétence. La question du sujet et de la relation à l'autre".

\section{La formation en alternance tendue entre deux pôles :}

Pour en venir à l'objet de ma contribution d'aujourd'hui, je suis reparti d'une réflexion sur l'alternance et sur les tensions qui traversent ces dispositifs de formation en alternance : en particulier ceux des métiers du secteur sanitaire et social.

Pour évoquer ces tensions, je me suis appuyé tout d'abord sur un document qui a été élaboré dans le cadre de la conférence de consensus sur l'alternance qui s'est déroulée en 2011 et 2012. Organisée par l'Institut Jacotot et le CNAM, elle a donné lieu à deux numéros de la revue Éducation Permanente en mars et en décembre 2012. Un certain nombre de questions ont été posées aux experts consultés pour cette conférence de consensus. Et la première de ces questions partait de l'idée selon laquelle l'alternance est productrice de tensions, notamment entre :

- lieu de travail (stages) / centre de formation

- temps, rythmes de travail / temps, rythmes de formation

- produire (travailler, rendre un service) / se construire (apprendre, se former)

- statut de stagiaire (professionnel en formation) / étudiant (élève)

- savoirs d'action / savoirs théoriques

La formation en alternance se caractériserait donc par cette tension entre une formation à dominante pratique, sur le terrain, et une formation «théorique », de type scolaire, en institut. Cette représentation binaire est bien évidemment réductrice, même si elle reste très présente dans les discours sur l'alternance. On peut caractériser ainsi chacun de ces deux pôles de la formation en alternance ainsi conçue (tableau 1) :

Tableau 1 : Une première tension entre les deux pôles de l'alternance

\begin{tabular}{|l|l|l|}
\hline Pôle & Pratique & "Théorique" \\
\hline lieu & lieu de travail, de stage & école, institut de formation \\
\hline méthode & "by doing, montrer, imiter & enseignement \\
\hline formateur & maître d'apprentissage, tuteur & professeur, enseignant \\
\hline
\end{tabular}




\begin{tabular}{|l|l|l|} 
fondé sur & $\begin{array}{l}\text { la tradition, les règles de l'art, } \\
\text { du métier }\end{array}$ & $\begin{array}{l}\text { les savoirs académiques, } \\
\text { la science }\end{array}$ \\
\hline résultats & savoir $(\mathrm{y})$ faire & savoirs, connaissances \\
\hline critères d'évaluation & $\begin{array}{l}\text { efficacité et conformité au } \\
\text { genre professionnel }\end{array}$ & $\begin{array}{l}\text { conformité aux savoirs } \\
\text { enseignés }\end{array}$ \\
\hline
\end{tabular}

Mais on peut aussi essayer de dépasser cette dichotomie. C'est ce que fait Guy Jobert dans son éditorial pour le numéro 193 de la revue Éducation Permanente, dont le titre est « Du discours à l'épreuve ». Guy Jobert revient sur cette opposition : d'un côté le discours, les discours, les théories, de l'autre l'épreuve du réel, du travail, de la pratique. Mais il ajoute aussitôt que ce débat entre théorie et pratique est dépassé, comme doit être dépassé celui entre savoir et comprendre. Il écrit : « pour comprendre, il faut en savoir plus (...) Quand on butte sur une difficulté pour comprendre, le moyen d'en sortir est d'apprendre plus ». Il va jusqu'à suggérer qu'il faut « plus d'école » signifiant par là qu'améliorer la formation en alternance ne doit pas reposer sur une augmentation du temps de stage, de l'activité pratique sur le terrain, mais sur un accroissement, ou un approfondissement des connaissances requises pour comprendre ce qui se joue sur le terrain, sur les lieux de travail. A ceux qui considèrent que l'essentiel des compétences s'acquièrent par l'action, il répond qu'il faut connaître et comprendre pour agir efficacement, pour dépasser les difficultés de l'action. Le discours et l'épreuve ne doivent plus être opposés : le discours sur l'épreuve, étayé par la connaissance, la compréhension des situations, permet de faire face à l'épreuve, de la surmonter, parce qu'il contribue à renforcer le pouvoir d'agir. La tension entre le discours "théorique" et l'épreuve de la pratique, leur opposition dialectique, serait dépassée par "le discours sur l'épreuve", un supplément de connaissance. Mais Jobert ne précise pas, dans ce texte, comment ce discours sur l'épreuve se construit, par quelle méthode, quel type de dispositif. Ailleurs, notamment dans son livre "Exister au travail" (2014), un chapitre aborde cette question. Il a pour titre : "méthodologie : la clinique, un certain régime de production des connaissances". (J'y reviendrai).

Mais, plutôt que de représenter cette problématique en termes d'opposition dialectique, et de dépassement de la contradiction ou de la tension entre les deux termes de l'opposition, je voudrais plutôt la reformuler, comme le thème du symposium nous y incite, en terme de reliance. Et faire référence à la dialogique d'Edgar Morin.

\section{Quelle reliance entre les deux pôles?}

Je rappelle tout d'abord une définition de la notion de reliance proposée par J-L. Le Moigne : "Cette faculté de l'esprit qui est d'articuler ce qui est séparé et de relier ce qui est disjoint, de 
distinguer des formes ou des 'patterns'sans pour autant les découper, et d'identifier les 'tiers'ou les 'liants' qui les interfacent, n'est-ce pas aussi cela que nous entendons maintenant par la faculté de reliance ?" (Le Moigne 2008).

Ceux qui ont à concevoir des dispositifs de formation en alternance ont bien à articuler ce qui est séparé et à mobiliser cette faculté de reliance s'ils prétendent que leur dispositif relève d'une alternance qui n'est pas simplement "juxtapositive", mais plutôt d'une alternance intégrative. Je me suis donc demandé : qu'est-ce qui remplit la fonction de tiers entre les deux pôles de l'alternance? Et, dans l'analyse des dispositifs de formation qui préparent aux métiers du secteur sanitaire et social, il me semble que l'on peut repérer deux approches de ce qui peut faire office de liant, approches qui correspondent à deux conceptions de la formation : une approche que l'on peut qualifier de "technique" et une approche clinique. La première repose sur l'utilisation de référentiels (référentiels métiers ou d'activités, référentiels de compétences, référentiels de formation...) et d'outils, de grilles d'évaluation de l'acquisition des compétences listées dans les référentiels. Elle se réfère à une certaine "approche par les compétences" aujourd'hui dominante dans ce domaine.

La deuxième repose sur des activités de formation se référant à une approche clinique. Elle met en œuvre un retour réflexif sur les situations vécues (notamment en stage) à travers des groupes d'analyse des pratiques et la production d'écrits, en particulier de mémoires professionnels. Ces deux types de dispositifs cohabitent dans les formations du secteur sanitaire et social, en proportion variable, ce qui crée parfois, en plus des tensions entre les deux pôles de l'alternance, un sentiment de paradoxe ressenti aussi bien chez les formateurs que chez les étudiants (Hébrard 2011).

Le schéma suivant donne une image de ces deux approches de la reliance entre les deux pôles de l'alternance. Il tente de représenter un modèle dialogique introduisant un peu de complexité dans la réflexion sur les tensions caractérisant l'alternance.

\section{Schéma 1 : Deux approches de la reliance}

approche clinique par l'analyse des pratiques

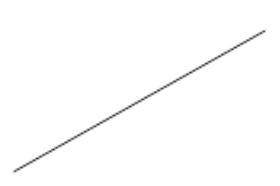

pôle « théorique »
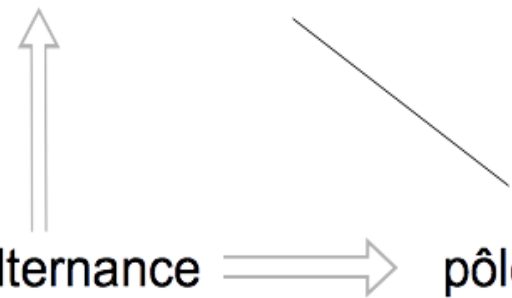

alternance pôle pratique
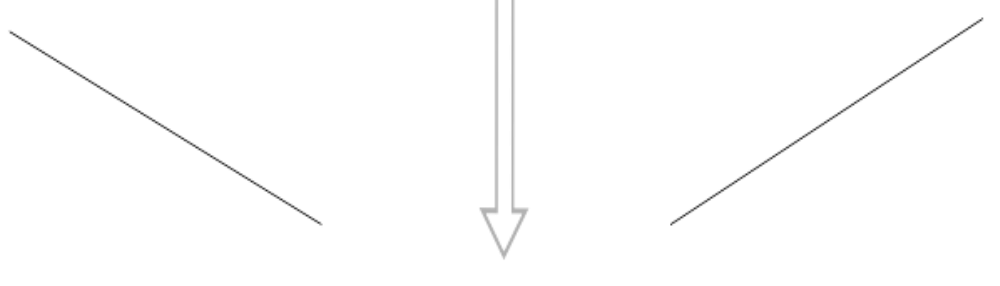
On peut alors se demander à quelles conditions chacune de ces approches, ou leur combinaison, peuvent effectivement produire de la reliance, et donc permettre aux personnes en formation de faire des liens entre ce qu'ils apprennent dans chacun des espaces et des temps de la formation en alternance. On peut également s'interroger sur les limites, les difficultés auxquelles ces approches risquent de se heurter, et qui peuvent alors faire obstacle à la reliance et rendre plus difficile l'intégration des différents apprentissages et la construction de la compétence professionnelle. Pour illustrer la forme que peut prendre une reliance qui fonctionne je prendrai l'exemple d'un dispositif de formation dans lequel, à la suite d'une période de stage pratique, les personnes en formation sont amenées à participer à un groupe d'analyse de pratiques. Puis il leur est demandé de rédiger un texte dans lequel ils doivent faire le récit d'une situation-problème rencontrée en stage et en faire l'analyse en mobilisant des concepts acquis au cours des enseignements théoriques reçus au centre de formation. Le référentiel est alors utilisé pour définir des critères d'évaluation de cet écrit, pour repérer les connaissances et compétences acquises et ce qui sera à acquérir ou à approfondir à l'occasion d'un prochain stage, d'un travail personnel ou de toute autre activité d'apprentissage. Des dispositifs de ce type se rencontrent dans la formation des infirmières et certains travailleurs sociaux français.

Mais quelles sont les conditions à remplir pour qu'un tel dispositif soit "reliant" et à quelles difficultés ce processus peut-il se heurter?

\section{Quelques conditions de la reliance ?}

Une première difficulté serait due à une séance d'exploitation de stage et/ou la rédaction de textes qui se limiteraient à faire le récit de situations sans problématisation ni véritable travail d'analyse (formulation d'hypothèses explicites, étayées par des concepts ou des modèles pertinents). Le passage par le haut du schéma n'aurait alors pas rempli sa fonction de reliance ; l'expérience pratique n'étant pas reliée aux savoirs conceptuels. Cette difficulté est plusieurs fois mentionnée dans les entretiens que nous avons menés auprès de formateurs-trices du secteur de la santé. 
Une autre difficulté est due à l'utilisation d'un référentiel comme une sorte de manuel ou de mode d'emploi, réduit à une liste d'opérations et de savoir faire sans aucune mention des connaissances, des concepts ou des modèles théoriques susceptibles d'éclairer les activités réalisées en stage. La compréhension des situations rencontrées dans la pratique risque alors d'être insuffisante (Jobert 2012) et se limiter à l'exécution de gestes dont la pertinence et la justesse ne seraient pas garanties. Cela soulève à la fois la question de la nature et du contenu des référentiels et celle de leur mode d'utilisation.

Une des difficultés dues à la nature des référentiels concerne les relations entre les formateurs des écoles ou instituts de formation et les professionnels qui encadrent les étudiants dans les lieux de stage et doivent évaluer le développement de leurs compétences. Ils doivent coopérer pour cela, mais certains des outils censés les y aider se révèlent à la fois lourds à manier (l'expression "d'usine à gaz" revient souvent) et peu pertinents pour évaluer certaines compétences du cœur de métier. Ils ont en effet du mal à prendre en compte les dimensions relationnelles de ces métiers. Les difficultés viennent de la formulation de certaines des compétences ou des indicateurs supposés permettre d'en mesurer l'acquisition. Ils sont abordés selon une approche réductrice, dans laquelle les relations éducatives ou le care sont réduits à la maîtrise d'un ensemble de techniques de communication ou de "gestion", par exemple de gestion de projet ou de gestion des conflits. (Hébrard 2015).

Si l'on considère le référentiel, et les autres "outils" mis en oeuvre, comme des instruments (au sens de Rabardel 1995), il faut retenir l'idée que :

"Le processus d'appropriation d'un artefact s'accompagne, chez le sujet, de constructions représentatives relatives à l'instrument, à la réalité sur laquelle il permet d'agir ou qu'il permet d'analyser". L'auteur ajoute que cette appropriation "s'accompagne aussi de l'élaboration de structures permettant l'organisation de l'action du sujet : les schèmes d'utilisation " qui constituent ensuite "une base stable pour son activité."

Lorsque les référentiels, comme nous l'avons montré pour certains d'entre eux (Hébrard 2013 a), sont élaborés sur la base d'une conception techniciste, sinon taylorienne de l'activité professionnelle, ils risquent d'induire une représentation réductrice de l'activité, très éloignée de la complexité du travail réel. Ils peuvent alors constituer un obstacle, plutôt qu'un instrument facilitant les liens entre les deux pôles de l'alternance, entre connaissances théoriques et activité pratique. De plus, lorsque l'approche "technique" est dominante, aux dépens de l'approche clinique cela contribue à construire une identité professionnelle "technicienne" dans des métiers où les compétences relationnelles et sociales sont au coeur de l'activité. En effet le dispositif de formation peut être considéré comme un "macro instrument" qui conditionne, chez les formateurs et les personnes en formation un ensemble 
de représentations et de schèmes d'action constituants de l'identité et de la professionnalité.

Plus globalement, ces référentiels se caractérisent souvent par le découpage de l'activité en une série de tâches et d'opérations élémentaires et la définition des compétences comme une série de savoir faire opératoires, formulés avec des verbes d'action, décrivant en fait des "performances". C'est le cas pour les métiers de la santé et certains métiers du travail social, notamment les moins qualifiés (jusqu'à 76 opérations pour les aides-soignants et 108 opérations pour les auxiliaires de puériculture)

Le tableau 2 ci-dessous présente, pour certains diplômes du travail social : Diplômes d'État d'Ingénierie Sociale, d'Éducateur Spécialisé et de Moniteur Éducateur (DEIS, DEES, DEME) et pour quelques diplômes paramédicaux : Infirmière de Bloc Opératoire, Infirmière Diplômée d'État et Auxiliaire de Puériculture (IBO, IDE et AP) le découpage du métier en activités, compétences et indicateurs de compétences selon les référentiels correspondants. 
Tableau 2 : Activités, compétences et indicateurs

\begin{tabular}{|l|c|c|c|}
\hline Diplômes & $\begin{array}{c}\text { activités } \\
\text { (opérations) }\end{array}$ & compétences & indicateurs \\
\hline DEIS & 35 & 10 & 28 \\
\hline DEES & 16 & 19 & 79 \\
\hline DEME & 40 & 12 & 47 \\
\hline IBO & 8 & 63 & 186 \\
\hline IDE & 9 & 77 & 117 \\
\hline AP & $8(108)$ & 56 & 60 \\
\hline
\end{tabular}

\section{Un regard critique des professionnels et des chercheurs}

Cette approche par les compétences trop souvent réductrice de la complexité, qui est devenue dominante dans les approches de l'ingénierie de la formation, a fait l'objet, depuis plusieurs années de nombreuses critiques (Stroobants 1993, 2002, Dolz et Ollagnier 2002, Jonnaert 2002, Crahay 2006, Gori 2011).

Si l'on regarde comment et par qui les référentiels sont conçus, on constate, le plus souvent, qu'ils sont établis par des instances constituées en majorité par les employeurs et les prescripteurs ou dans lesquelles ils jouent un rôle dominant ${ }^{1}$. Ces instances, assistés d'experts en "réingénierie de formation", conçoivent des référentiels métiers essentiellement à partir de ce que les ergonomes appellent le travail prescrit, par opposition au travail réel - même s'il leur arrive d'évoquer ce dernier (Coudray et Gay 2009).

C'est d'ailleurs aussi contre cette approche et cette méthode que protestent actuellement plusieurs organisations de travailleurs sociaux, et un collectif de formateurs et d'enseignants-chercheurs. Une première pétition intitulée "travail social : participons à l'avenir de nos métiers" ${ }^{2}$ réagissait contre un rapport intitulé « Métiers et complémentarités - architecture des diplômes en travail social », validé par la Commission Professionnelle Consultative (CPC), en décembre dernier (par 24 voix pour, 6 voix contre, celles de la CGT, CFDT et FO et 1 abstention : la CFTC).

Plus récemment, un article et un appel à la signature émanant d'un collectif d'universitaires, de formateurs et de chercheurs : " Défendre les métiers sociaux" est paru dans Le Monde

1 Dans la composition de la Commission Professionnelle Consultative du travail social, les représentants des salariés sont 10 sur 40)

2 http://www.petitions24.net/read/17070/22291868 
Au-delà de la défense des professions existantes ou d'une certaine conception de ces professions, que ce projet de réforme prétend "refonder", c'est la ré-ingénierie des formations qu'ils critiquent et à laquelle ils opposent une vision du travail social et de la formation des travailleurs sociaux, fondée sur l'activité réelle qu'ils conduisent auprès des personnes en difficultés ou malades.

De quoi ce conflit est-il un symptôme supplémentaire ? D'une opposition entre deux conceptions des métiers du travail social et de la formation des travailleurs sociaux. L'une est fondée sur une description fine des fonctions, activités, tâches, voire opérations constituant le travail prescrit, consignée dans les référentiels métiers, puis traduite en termes de référentiels de compétences. Ces référentiels de compétences servent ensuite à élaborer des grilles et autres outils d'évaluation de la maîtrise des compétences listées, à l'aide d'une série de critères et d'indicateurs formulés en termes de performances.

Ceux qui critiquent cette ingénierie de formation et cette conception de la professionnalité des travailleurs sociaux et du personnel paramédical (et j'en fais partie) lui reprochent son approche techniciste de ces métiers et sa conception réductrice, sinon behavioriste de la formation (Hébrard 2013 b). Elle conduit à une représentation de l'identité professionnelle des travailleurs sociaux et des personnels de santé que l'on peut résumer par l'expression d'identité technicienne. Elle s'accompagne d'un développement des procédures et protocoles formalisés et normatifs qui conduisent à un déprofessionnalisation et à une remise en cause de l'autonomie de ces professionnels et du cœur de leur métier qui est lié à la relation singulière au contact des "usagers". Ces réformes sont vécues comme des tentatives de déqualification par ceux qui exercent au quotidien ce travail exigeant et difficile, et une méconnaissance de leur activité réelle. Ils lui opposent une identité clinicienne, fondée sur des connaissances, mais aussi sur une éthique professionnelle qui se traduit par une posture acquise par l'expérience et transmise par les professionnels qui encadrent les étudiants en stage. ${ }^{4}$

\section{Perspectives :}

Nous continuons à travailler sur cette question, en particulier à travers deux études l'une portant sur

3 http://www.lemonde.fr/idees/article/2015/06/23/defendre-les-metiers-sociaux_4660307_3232.html

4 J'ai ailleurs (Hébrard 2011, 2013a) indiqué sur quelles références peut se fonder cette approche clinique des métiers de la relation ainsi que de la formation préparant à ces métiers. (Cifali et Giust-Desprairies 2008, en particulier les chapitres rédigés par Mireille Cifali et par Françoise Bréant). 
la conduite des séances d'analyse des pratiques et l'autre sur l'accompagnement des étudiants dans l'élaboration de mémoires et autres écrits à distance (Vallet et Griffith 2013) ou en présence. Nous mobilisons pour cela le type de méthodes utilisées par Guy Jobert dans l'ouvrage déjà cité. Elles combinent des groupes de travail et des entretiens individuels permettant de recueillir les points de vue des personnes directement concernées par ces dispositifs de formation, leur vécu de ces activités et de situations singulières rencontrées, le sens qu'ils attribuent à leur travail et au rôle qu'ils y jouent. Nous considérons qu'il s'agit de recherches-actions qui ont un double objectif : d'une part (je cite Jobert 2014) "la production discursive des agents est destinée à nourrir un travail d'élaboration intellectuelle susceptible d'élever le niveau de compréhension du "monde vécu des agents" d'abord pour eux-mêmes (p. 63) ; d'autre part, du fait de "l'interaction heuristique des participants avec les chercheurs", se constitue une sorte de "communauté scientifique élargie" dont la pensée est alimentée par les "concepts quotidiens" ou pragmatiques des acteurs et par les concepts scientifiques ou théoriques apportés par les chercheurs (p. 65). Les interprétations formulées font l'objet de discussion et sont doublement validées : d'une part par les participants aux groupes de travail et les personnes interviewées et d'autre part par la communauté des chercheurs à commencer par les membres de l'équipe de recherche.

\section{Bibliographie :}

Bouyssières, P. (2011). "Savoirs formatifs » et genres professionnels chez les formateurs en formation à l'université : étude des résultats de recherches-actions. Communication au colloque : «Université et métiers de la formation : quels enjeux, quelles spécificités? »Université Toulouse II-le Mirail

Cifali M. et Giust-Desprairies F. (2008.). Formation clinique et travail de la pensée. Bruxelles : De Boeck

Collectif (2015). "Travail social : participons à l'avenir de nos métiers" récupéré en juin 2015 sur http://www.petitions24.net/read/17070/22291868

Collectif (2015). " Défendre les métiers sociaux" Le Monde (23.06.2015).

http://defendrelesmetierssociaux.com/

http://www.lemonde.fr/idees/article/2015/06/23/defendre-les-metiers-sociaux_4660307_3232.html

Coudray, M.A. et Gay, C. Le défi des compétences. Comprendre et mettre en auvre la réforme des études infirmières. Issy-les-Moulineaux : Elsevier-Masson, 2009.

Crahay, M. Dangers, incertitudes et incomplétude de la logique de la compétence en éducation, Revue Française de Pédagogie, 2006, № 154, pp.97-110.

Dolz, J et Ollagnier, E. (Dir.). L'énigme de la compétence en éducation, Bruxelles : De Boeck Université, 2002.

Gori, R. (2011). La dignité de penser. Les liens qui libèrent.

Hébrard, P. (2011). L'humanité comme compétence ? Une zone d'ombre dans la professionnalisation aux métiers de l'interaction avec autrui. Les Sciences de l'Éducation. Pour 
l'ère nouvelle. Vol. 44, n², 103-121.

Hébrard, P. (2013a). Quelle « approche par les compétences » et quels référentiels pour la formation professionnelle aux métiers de la relation humaine ? Les Dossiers des Sciences de l'Éducation, $\mathrm{n}^{\circ}$ 30, 17-34. Toulouse : P.U. du Mirail.

Hébrard, P. (2013b). Ambiguities and paradoxes in a competence-based approach to vocational education and training in France. European Journal for Research on the Education and Learning of Adults, $\quad$ RELA, vol. $\quad 4, \quad \mathrm{n}^{\circ} 2, \quad 111-127 . \quad$ En ligne : http://www.rela.ep.liu.se/contents.asp?doi=10.3384/rela.2000-7426.201342

Hébrard, P. (2015). Compétence et travail du care dans la formation aux métiers de la relation humaine, in V. Haberey et J-L. Heeb : Pour une critique de la compétence. La question du sujet et de la relation à l'autre. Paris : L'Harmattan.

Jobert, G. (2014). Exister au travail. Les hommes du nucléaire. Toulouse : Éres.

Jonnaert, P. (2002). Compétences et socioconstructivisme. Un cadre théorique. De Boeck.

Le Moigne J-L. (2008). Edgar Morin, le génie de la Reliance, Synergies Monde, n 4, 177-184.

Stroobants, M. (2002). La qualification ou comment s'en débarrasser, in : Dolz, J et Ollagnier, E. (Dir.). L'énigme de la compétence en éducation, Bruxelles : De Boeck Université.

Vallet, P. et Griffith, O. (2013). Comment les formateurs accompagnent le développement de l'autonomie des apprenants dans les dispositifs "en partie" à distance ? Biennale UNAFORIS : Les formations sociales en transformation : l'affaire de tous ? St Ouen, novembre 2013. 